\title{
Indicador de Salubridade Ambiental de municípios lindeiros e não lindeiros da Bacia Hidrográfica do Paraná III
}

\author{
Indicator of Environmental Health the surrounding cities and not bordering of \\ the Itaipu Paraná Basin III
}

\author{
Ana Claudia Cabral Mari' \\ Luana Patrícia Pinto²(*) \\ Alvaro Mari Junior ${ }^{3}$ \\ Lucas dos Santos Dierings ${ }^{4}$ \\ Elisandro Pires Frigo ${ }^{5}$
}

\section{Resumo}

O saneamento ambiental contempla ações para a sociedade e meio ambiente, como o abastecimento de água, coleta e disposição de resíduos, drenagem urbana e também o controle de doenças. Devido à sua importância, surgiram ferramentas para auxiliar na execução das atividades de controle e fiscalização urbana, sendo uma delas, o indicador de salubridade ambiental (ISA). O ISA é calculado a partir de fatores relacionados às práticas do saneamento, cujos valores baixos encontrados devem-se à devem ser analisados isoladamente. O objetivo deste estudo foi calcular o Indicador de Salubridade Ambiental para municípios lindeiros e não lindeiros estudados e levantar dados de consumo médio de energia elétrica e água, verificando se há uma relação entre ambas, e se os royalties promovem benefícios diretos aos municípios lindeiros. Os dados utilizados para o cálculo do ISA foram adquiridos por levantamentos a campo e pesquisas em bancos de dados. Os resultados obtidos representam a salubridade de cada uma das áreas estudadas. Como resultado, neste trabalho, foi possível definir que a maioria dos municípios lindeiros e não lindeiros da bacia hidrográfica do Paraná III, encontram-se em condições salubres, contudo, algumas medidas devem ser tomadas para que seus parâmetros se mantenham apropriados para o desenvolvimento econômico e social.

1 Dr.; Agronomia; Universidade Estadual Paulista Júlio de Mesquita Filho, UNESP, Brasil; Endereço: Av. Prof. Mário Rubens Guimarães Montenegro, s/n - Campus de Botucatu, Botucatu - SP, CEP: 18618-687; E-mail: ana_c_cacau@ hotmail.com $\left({ }^{*}\right)$ Autor para correspondências

2 Mestre; Engenharia Agrícola na área de concentração de recursos hídricos e saneamento ambiental; Universidade Estadual do Oeste do Paraná, UNIOESTE, Brasil; Assistente de Laboratório e Pesquisa na Empresa SCHEFFER; Endereço: Av. André Antônio Maggi, 2009 - Centro, Sapezal - MT, CEP: 78365-000; E-mail: luana.kozak@gmail.com

3 Dr.; Agronomia; Universidade Estadual Paulista Júlio de Mesquita Filho, UNESP, Brasil; Endereço: Av. Prof. Mário Rubens Guimarães Montenegro, s/n - Campus de Botucatu, Botucatu - SP, CEP: 18618-687; E-mail: professor.alvaro. mari@gmail.com

4 Graduando; Agronomia; Universidade Federal do Paraná, UFPR, Setor Palotina, Brasil; Pesquisador no grupo de pesquisa Uso e Manejo Sustentável de Água e Solo; Endereço: Rua Pioneiro, 2153, Jardim Dallas, CEP: 85950-000, Palotina, Brasil; E-mail: lucasdierings@live.com

5 Dr.; Irrigação e Drenagem; Faculdade de Ciências Agronômicas - UNESP, FCA, Brasil; Professor Associado do Setor Palotina da Universidade Federal do Paraná, Coordenador de Ensino Superior da Secretária da Ciência, Tecnologia e Ensino Superior do Paraná; Endereço: Rua Pioneiro 2153, Jardim Dallas. CEP: 85950-000, Palotina, PR, Brasil; E-mail: epfrigo@gmail.com

\begin{tabular}{llllll}
\hline Ambiência & Guarapuava (PR) & v.l5 n.I & p.57 - 72 & Jan/Abr 20I9 & ISSN I808 - 025I
\end{tabular}


Também é possível concluir que, nos municípios lindeiros estudados, os royalties não têm impacto significativo. O ISA apresentou-se como uma ótima ferramenta para definição da salubridade ambiental dos municípios em estudo com potencial de uso para monitoramento e de gestão ambiental.

Palavras-Chave: índices municipais; saúde ambiental; socioeconômico.

\section{Abstract}

Environmental sanitation includes actions for society and the environment, such as water supply, collection and disposal of waste, urban drainage and also disease control. Because of it's importance, there were tools to assist in the implementation of urban control and inspection activities were created, and the environmental health indicator (EHI) was created as one of these tools. EHI is calculated using data on some factors, all related to sanitation practices that should be analyzed in isolation. The objective of this study was to calculate the Environmental Health Indicator for the surrounding and non-mountained municipalities studied and to collect data on average consumtion of electric energy and water, verifying if there is a relation between them, and if the royalties promote direct benefits to the surrounding municipalities. The data used to calculate the ISA were acquired by field surveys and surveys in databases, the results obtained represent the salubrity of the Each of the studied areas. As a result of this work, it was possible to define that most of the coastal and non-coastal municipalities of the Paraná III watershed are in healthy conditions, however, some measures must be taken so that their parameters remain appropriate for economic development and social. It is also possible to conclude that, in the municipalities studied, the royalties, don't have a significant impact. The ISA presented as a great tool to define the environmental health of municipalities studies with potential use for monitoring and environmental management.

Key words: municipal indexes; environmental health; socialeconomic.

\section{Introdução}

Nos dias atuais, o crescimento populacional progride de forma desuniforme, mesmo dentro de pequenos limites geográficos, como um município. De certa forma isso pode ser justificado pela maneira diferenciada com a qual cada região é gerenciada. De acordo com Reis e Oliveira (2011), o fenômeno globalização que se alastrou nas últimas décadas, intensificou a desigualdade social e a insustentabilidade da relação homem e natureza. Observando tal problema, alguns estudos buscam formas de detectar estas disparidades e, com base nelas, permitir ações pontuais que melhorem as condições de vida tornando-as igualitárias. O Indicador de Salubridade Ambiental - ISA é um exemplo (AKERMAN et al., 1994).

A salubridade ambiental é o estado de saúde em que vive a população sendo ela urbana ou rural, e é influenciada pela sua capacidade de impedir ou prevenir a ocorrência de endemias ou epidemias (FUNASA, 2006). O ISA é um indicador que foi desenvolvido pelo CONESAN, e basicamente, busca estabelecer índices para solucionar os problemas relacionados ao saneamento básico de cada área (bairro, vila, município ou estado). Esta ferramenta é considerada extremamente 
importante, pois permite ser adaptada aos diferentes problemas específicos de cada local, sendo adequada para estudar problemas referentes à coleta de resíduos, esgoto sanitário, abastecimento de água, recursos hídricos, doenças, educação e fatores socioeconômicos, permitindo diagnosticar tais problemas (PIZA, 2000).

Além disso, através de análises o ISA consegue apontar quais os pontos que devem ser atendidos com mais urgência. Por meio da análise do ISA, torna-se possível reavaliar os pontos nos quais estão sendo gastos os fundos municipais, visando não só o redirecionamento das verbas, mas também a redução de gastos pelo seu uso inadequado em pontos de menor necessidade (WHO, 2014).

Referindo-se às características do ISA, este apresenta indicadores relacionados aos corpos hídricos (que no Brasil é principal fonte de geração de energia elétrica) que podem constatar, por exemplo, o fato de que a quantidade de água potável em relação à população vem diminuindo. Além disso, pode ser utilizado para levantar dados referentes a alfabetização e às características socioeconômicas, gerando a possibilidade de estudar a sua relação, e o consumo de água e energia elétrica (SANTOS et al., 2015).

Devido à importância em analisar problemas associados ao saneamento básico, bem como ao desperdício de água e de energia elétrica, torna-se necessário um estudo que consiga verificar se existe alguma característica, em uma sociedade, que seja responsável por tais problemas. Desta forma, o trabalho tem como proposta de, através da verificação das taxas de alfabetização e das características socioeconômicas, detectar se estas podem ser consideradas causadoras dos problemas citados (PINTO et al., 2016).

Os municípios atingidos pelo alagamento de terras para a formação do reservatório da Itaipu (denominados lindeiros ao lago) recebem royalties, devido ao fato de que a maior parte dessas áreas alagadas seriam agricultáveis. $O$ repasse do valor a ser pago a título de royalties pela Itaipu Binacional, varia conforme a geração de energia destinada a comercialização em cada mês. São 15 municípios beneficiados, porém no presente trabalho foram dados de apenas oito municípios (LEISMANN, 2006).

Para Leismann (2006), os royalties têm a finalidade de promover o desenvolvimento socioeconômico dos municípios, devendo assim proporcionar melhor qualidade de vida aos habitantes. Com base nisso, o trabalho buscou gerar dados comparativos entre os municípios que recebem e os que não recebem este benefício, para verificar se o principal objetivo dos royalties está sendo atingido.

Sendo assim, o objetivo deste estudo foi calcular o Indicador de Salubridade Ambiental para municípios lindeiros e não lindeiros estudados e levantar dados de consumo médio de energia elétrica e água, verificando se há uma relação entre ambas, e se os royalties promovem benefícios diretos aos municípios lindeiros

\section{Material e Métodos}

O Estado do Paraná localiza-se na região Sul do Brasil, faz divisa com os estados do Mato Grosso do Sul a Noroeste, Santa Catarina ao Sul e com o Estado São Paulo a Norte e a Leste. Os municípios estudados estão localizados no Oeste do Paraná, na bacia hidrográfica do Paraná III (Figura 1). 


\section{Figura 1 - Localização dos municípios lindeiros e não lindeiros estudados da Bacia do Paraná III, PR}

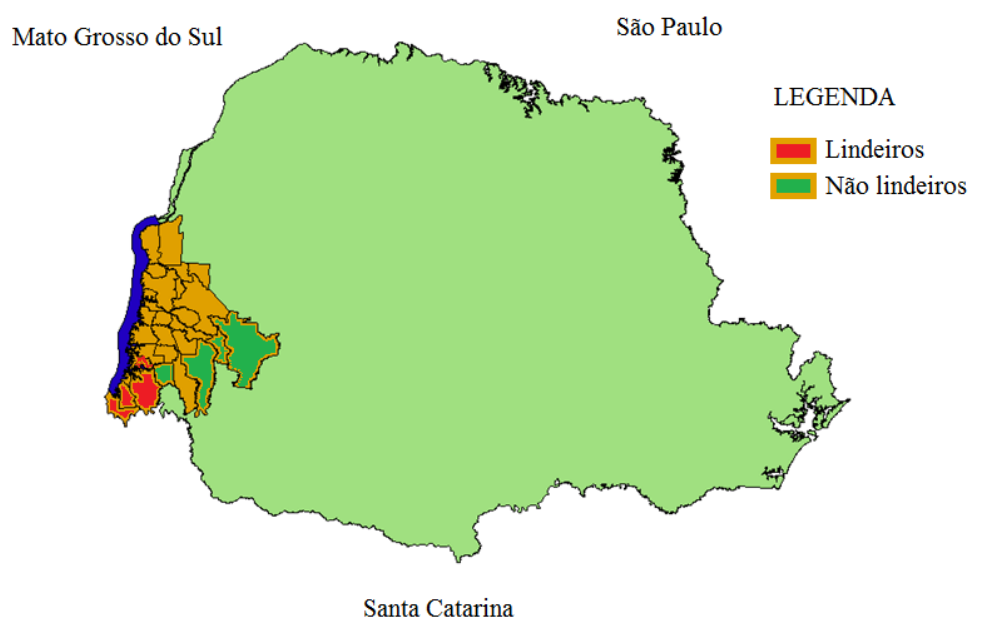

A Bacia do Paraná III, conhecida como BP III, é ocupada por vinte e nove municípios, que perfazem o total aproximado de um milhão de habitantes, sendo estes municípios: Mundo Novo, Altônia, Guaíra, Terra Roxa, Mercedes, Nova Santa Rosa, Maripá, Marechal Cândido Rondon, Quatro Pontes, Pato Bragado, Entre Rio do Oeste, São Jose das Palmeiras, Ouro Verde, Toledo, Santa Helena, Diamante do Oeste, São Pedro do Iguaçu, Missal, Ramilândia, Vera Cruz do Oeste, Cascavel, Itaipulândia, Medianeira, Céu Azul, Matelândia, São Miguel do Iguaçu, Santa Terezinha de Itaipu, Foz do Iguaçu e Santa Tereza do Oeste.

O trabalho utiliza dados de oito destes municípios, sendo quatro municípios lindeiros ao lago de Itaipu, entre eles encontram-se Foz do Iguaçu, Santa Terezinha de Itaipu, São Miguel do Iguaçu e Itaipulândia. Já os municípios não lindeiros são Matelândia, Medianeira, Céu Azul e Cascavel. A escolha dos municípios se baseou na metodologia de Piza (2000), na qual alguns dos indicadores apoiam-se em um cálculo que se utiliza de quartis, tornando-se viável a utilização de um número de municípios múltiplo de quatro. A Tabela 1 mostra, de forma sucinta e organizada, os dados que foram utilizados neste trabalho. Além disso, estes índices são facilmente identificados e analisados a seguir (Tabela 2).

\section{Tabela 1 - Dados dos municípios em estudo.}

\begin{tabular}{ccccc}
\hline Municípios & $\begin{array}{c}\text { Hab. } \\
\text { Totais }\end{array}$ & $\begin{array}{c}\text { Hab. da } \\
\text { zona rural }\end{array}$ & $\begin{array}{c}\text { Área } \\
\text { territorial } \\
\text { aproximada } \\
\mathbf{( \mathbf { K m } ^ { 2 } )}\end{array}$ & $\begin{array}{c}\text { Unidades de } \\
\text { empresas atuantes } \\
\text { no setor do } \\
\text { comércio }\end{array}$ \\
\hline Foz do Iguaçu & 256.088 & 2.126 & 617 & 9.152 \\
Santa Terezinha de Itaipu & 22.127 & 2.004 & 259 & 818 \\
São Miguel do Iguaçu & 25.769 & 9.284 & 848 & 1.020 \\
Itaipulândia & 9.026 & 4.285 & 327 & 396 \\
Matelândia & 16.078 & 4.465 & 639 & 537 \\
Medianeira & 41.817 & 4.427 & 328 & 1.874 \\
Céu Azul & 11.032 & 2.645 & 1.179 & 336 \\
Cascavel & 286.205 & 16.156 & 2.100 & 13.063 \\
\hline
\end{tabular}


O ISA - Indicador de Salubridade Ambiental é calculado pela média ponderada de indicadores específicos da Equação 1.

$\mathrm{ISA}=\left(0,25 * \mathrm{I}_{\mathrm{AB}}\right)+\left(0,25 * \mathrm{I}_{\mathrm{ES}}\right)+\left(0,25 * \mathrm{I}_{\mathrm{RS}}\right)+\left(0,10 * \mathrm{I}_{\mathrm{CV}}\right)+\left(0,10 * \mathrm{I}_{\mathrm{RH}}\right)+\left(0,05 * \mathrm{I}_{\mathrm{SE}}\right)$

Onde:

$\mathrm{I}_{\mathrm{AB}}$ - Indicador de Abastecimento de Água

$\mathrm{I}_{\mathrm{ES}}$ - Indicador de Esgotos Sanitário

$\mathrm{I}_{\mathrm{RS}}$ - Indicador de resíduos Sólidos

$\mathrm{I}_{\mathrm{CV}}$ - Indicador de Controle de Vetores

$\mathrm{I}_{\mathrm{RH}}$ - Indicador de Recursos Hídricos

$\mathrm{I}_{\mathrm{SE}}$ - Indicador Sócio - Econômico.

\section{Tabela 2 - Descrição dos Indicadores de Salubridade Ambiental.}

\begin{tabular}{|c|c|}
\hline Indicador & Descrição \\
\hline $\begin{array}{l}\text { Indicador de abastecimento de água } \\
\text { (IAB): }\end{array}$ & $\begin{array}{l}\text { É obtido da média aritmética dos indicadores de } \\
\text { subindicadores: Indicador de Cobertura e Abastecimento } \\
\left(\mathrm{I}_{\mathrm{CA}}\right) \text {, Indicador de Qualidade da Água distribuída }\left(\mathrm{I}_{\mathrm{OA}}\right) \\
\text { e Indicador de Saturação do Sistema Produtor }\left(\mathrm{I}_{\mathrm{SA}}\right) \text {. Ele } \\
\text { visa quantificar os domicílios atendidos pelo sistema de } \\
\text { abastecimento de água. }\end{array}$ \\
\hline Indicador de esgoto sanitário (IES): & $\begin{array}{l}\text { Se dá pela média aritmética de seus subindicadores: } \\
\text { Indicador de Cobertura em Coleta de Esgotos e Tanques } \\
\text { Sépticos }\left(\mathrm{I}_{\mathrm{CE}}\right) \text {, Indicador de Esgotos Tratados e Tanques } \\
\text { Sépticos }\left(\mathrm{I}_{\mathrm{TE}}\right) \text { e Indicador de Saturação do Tratamento } \\
\left(\mathrm{I}_{\mathrm{SE}}\right) \text {. Visa quantificar os domicílios atendidos por redes } \\
\text { de esgoto, indicar a carga poluidora. }\end{array}$ \\
\hline Indicador de resíduos sólidos (IRS): & $\begin{array}{l}\text { É obtido através da média aritmética dos subindicadores: } \\
\text { Indicador de coleta de lixo }\left(\mathrm{I}_{\mathrm{CR}}\right) \text {, Indicador de Tratamento } \\
\text { e Disposição Final }\left(\mathrm{I}_{\mathrm{QR}}\right) \text { e Indicador de Saturação do } \\
\text { tratamento e disposição final de resíduos sólidos }\left(\mathrm{I}_{\mathrm{SR}}\right) \text {. } \\
\text { Visa quantificar domicílios que são atendidos pela coleta } \\
\text { seletiva. }\end{array}$ \\
\hline Indicador de controle de vetores (IVC): & $\begin{array}{l}\text { São considerados insetos ou animais que são usados } \\
\text { como veículos para infecção. Para realizar esse cálculo, é } \\
\text { feita a média ponderada entre os indicadores de Dengue, } \\
\text { Esquistossomose e Leptospirose, visando identificar a } \\
\text { necessidade de programas de incentivo de eliminação do } \\
\text { vetor. }\end{array}$ \\
\hline
\end{tabular}


É calculado pela média aritmética entre os Indicadores Indicador de riscos de recursos hídricos $\mathrm{I}_{\mathrm{OB}}$ que é a qualidade de água, $\mathrm{I}_{\mathrm{DM}}$ sendo a disponibilidade (IRH): dos mananciais e $\mathrm{I}_{\mathrm{FI}}$ identificação das fontes isoladas

Calculado a partir da média aritmética entre os Indicadores Indicador Sócio Econômico $\left(\mathrm{I}_{\mathrm{SE}}\right)$ de Renda $\left(\mathrm{I}_{\mathrm{RF}}\right)$, de Saúde Pública $\left(\mathrm{I}_{\mathrm{SP}}\right)$ e de Educação $\left(\mathrm{I}_{\mathrm{ED}}\right)$. Ele tem como objetivo destacar as características de uma população voltada à educação, mortalidade e renda.

Fonte: PIZA (2000).

Considerando sobre a ponderação e pontuação dos indicadores, Piza (2000), afirma que os valores obtidos pelos indicadores são pontuados em uma escala que varia de 0 (zero) a 100 (cem). No geral a pontuação se realizará da seguinte maneira: existe risco de vida (zero ponto) e se não existir risco de vida (100 pontos), esse critério se encontra logo abaixo (Tabela 3).

\section{Tabela 3 - Classificação dos Indicadores de Salubridade Ambiental.}

\begin{tabular}{cc}
\hline Condição de Salubridade & Pontuação do ISA \\
\hline Insalubre & $0-25,50$ \\
Baixa Salubridade & $25,51-50,50$ \\
Média Salubridade & $50,51-75,50$ \\
Salubre & $75,51-100,00$ \\
\hline
\end{tabular}

Fonte: SILVA (2006).

Os indicadores são compostos por entradas de dados de 5 anos. Assim, os dados utilizados neste trabalho são os referentes ao intervalo de 2008 a 2012.

As informações encontradas e fornecidas foram diferentes para o consumo de energia e água, pois, nos dados de abastecimento de água, foram fornecidas as quantidades de ligações anuais, não sendo possível separar estes dados em setores, ao contrário do que acontece no consumo de energia. Assim as informações referentes à energia elétrica e abastecimento de água dos últimos 5 anos foram calculados de modo que demonstrasse os dados por habitante, assim transformando os dados de uma maneira igualitária para posterior comparação entre as cidades.

Para o presente estudo foi utilizada a estatística descritiva, que teve por objetivo resumir as principais características dos conjuntos de dados por meio de tabelas. Por fim, as análises, tanto para o consumo de energia elétrica quanto para o abastecimento de água, foram realizadas com o auxílio do programa Assistat. O teste de média realizado foi o Teste de Tukey a 5\% de probabilidade, para verificar o grau de significância ou de diferença, verificando assim se estatisticamente existem diferenças entre os dados de consumo.

\section{Resultados e Discussão}

A Tabela 4 apresenta os resultados encontrados para os indicadores de primeira ordem e para o ISA de cada município estudado, sendo possível observar que a maioria dos valores encontrados foram considerados como "salubres". 
Tabela 4 - Resultado dos cálculos do ISA e de seus indicadores, 2008 a 2012

\begin{tabular}{|c|c|c|c|c|c|c|c|c|}
\hline \multirow[b]{2}{*}{ Municípios } & \multicolumn{7}{|c|}{ Indicadores / Pesos } & \multirow[b]{2}{*}{ Salubridade } \\
\hline & $\begin{array}{c}I_{\mathrm{AB}} / \\
(\mathbf{0 , 2 5})\end{array}$ & $\begin{array}{c}I_{\mathrm{ES}} / \\
(\mathbf{0 , 2 5})\end{array}$ & $\begin{array}{c}\mathrm{I}_{\mathrm{RS}}{ }^{\prime} \\
(\mathbf{0 , 2 5})\end{array}$ & $\begin{array}{c}I_{\mathrm{Cv}} / \\
(\mathbf{0 , 1 0})\end{array}$ & $\begin{array}{c}\mathrm{I}_{\mathrm{RH}} / \\
(\mathbf{0 , 1 0 )}\end{array}$ & $\begin{array}{c}\mathbf{I}_{\mathrm{SE}} / \\
(\mathbf{0 , 0 5})\end{array}$ & ISA & \\
\hline \multicolumn{9}{|c|}{ Municípios lindeiros ao lago de Itaipu } \\
\hline $\begin{array}{l}\text { Foz do } \\
\text { Iguaçu }\end{array}$ & 91,66 & 85,56 & 83,6 & 18,75 & 75,00 & 9,3 & 79,23 & Salubre \\
\hline $\begin{array}{c}\text { Santa } \\
\text { Terezinha } \\
\text { de Itaipu }\end{array}$ & 99,85 & 50,34 & 60,00 & 81,25 & 75,00 & 67,33 & 71,53 & $\begin{array}{c}\text { Média } \\
\text { Salubridade }\end{array}$ \\
\hline $\begin{array}{l}\text { São Miguel } \\
\text { do Iguaçu }\end{array}$ & 91,66 & 44,71 & 50,00 & 81,25 & 75,00 & 52,00 & 64,89 & $\begin{array}{c}\text { Média } \\
\text { Salubridade }\end{array}$ \\
\hline Itai & 98,59 & 48,79 & 96,66 & 43,75 & 75,00 & 84,00 & 77,08 & \\
\hline \multicolumn{9}{|c|}{ Municípios não lindeiros ao lago de Itaipu } \\
\hline Matelândia & 93,88 & 50,29 & 91,82 & 43,75 & 75,00 & 84,66 & 71,30 & $\begin{array}{c}\text { Média } \\
\text { Salubridade }\end{array}$ \\
\hline Medianeira & 100 & 37,67 & 93,33 & 81,25 & 75,00 & 26,00 & 74,66 & Salubre \\
\hline Céu Azul & 95,42 & 96,75 & 48,33 & 75,00 & 75,00 & 80,66 & 79,15 & Salubre \\
\hline Cascavel & 100 & 75,18 & 91,66 & 43,75 & 75,00 & 10,00 & 79,08 & Salubre \\
\hline
\end{tabular}

Embora alguns dos indicadores dos municípios tabelados como "Salubre" apresentem valores extremamente baixos, a classe destes municípios não foi reduzida devido ao peso maior dos sub-indicadores nos quais foram obtidos valores maiores. O contrário aconteceu com municípios que tiveram valores maiores em indicadores de menor peso. Ou seja, devido ao fato de que os indicadores: $\mathrm{I}_{\mathrm{AB}}, \mathrm{I}_{\mathrm{ES}}$ e $\mathrm{I}_{\mathrm{RS}}$ possuírem maiores pesos, estes são os principais responsáveis pela classe de salubridade neste trabalho.

\section{Foz do Iguaçu}

O município de Foz do Iguaçu obteve este valor em relação à $\mathrm{I}_{\mathrm{AB}}$, pois a empresa responsável consegue suprir a demanda do município, além de fornecer água com qualidade adequada. Cabe citar também, que por se tratar da mesma empresa responsável, os padrões são mantidos em todos os demais municípios estudados e, desta forma, os valores encontrados neste indicador, encontram-se todos em uma faixa de 90 a 100 pontos.

$\mathrm{O}$ valor encontrado para o $\mathrm{I}_{\mathrm{ES}}$ se deu pelo nível de atendimento da rede coletora de esgoto, que nos anos estudados foi de aproximadamente 80\%. A empresa afirma que 100\% do volume coletado de esgoto é tratado, no entanto, afirmam que existe uma problemática que se dá pela forma em que o esgoto é tratado nas Estações de Tratamento de Esgoto - ETE. 
O tratamento utiliza do sistema "RALF" (Reator Anaeróbio de Leito Fluidizado) que é extremamente prejudicado pela contaminação das redes coletoras, causada muitas vezes por ligações clandestinas, seja de águas pluviais de residências ou das redes públicas de galerias de águas pluviais.

O problema se agrava ainda mais quando acontece uma chuva de média ou de grande intensidade, pois o manto de substrato biológico (indispensável para o tratamento) é "lavado" e flui das ETE's, fazendo com que a capacidade de tratamento seja reduzida, levando um período de, aproximadamente, 60 dias no verão e 90 dias no inverno, para que haja a recuperação deste substrato. Estas informações foram cedidas pela Companhia de Saneamento do Paraná - Sanepar.

$\mathrm{O}$ valor para o indicador de vetores se deve à infestação por Aedes Aegypti, dado que no intervalo de 2008 a 2012 houveram aproximadamente 11.000 casos de dengue confirmados no município de Foz do Iguaçu, os responsáveis vêm buscando medidas para reduzir estes números. A explicação para o valor do $\mathrm{I}_{\mathrm{RH}}$ se dá pela existência do lago de Itaipu, que é utilizado para abastecimento público, além de contar com outros corpos hídricos. Já o valor alto do $\mathrm{I}_{\mathrm{RS}}$ se deu devido à existência de uma empresa especializada que consegue suprir completamente a demanda de coleta de resíduos para a população.

De acordo com a prefeitura do município, a economia da cidade está relacionada ao turismo e ao comércio atacadista, porém, para o $\mathrm{I}_{\mathrm{RH}}$ foi encontrado um baixo valor que está correlacionado à renda, à saúde e à educação, e não com as atividades primárias do município.

\section{Santa Terezinha de Itaipu}

Pode-se destacar o $\mathrm{I}_{\mathrm{ES}}$, que foi um baixo valor, e isso ocorre devido ao fato de que apenas 2.823 domicílios são atendidos pela coleta e tratamento de esgoto, pois no município, existem 7.061 imóveis, ou seja, menos de 40\% da população é atendida. O valor para o $\mathrm{I}_{\mathrm{RS}}$ se fundamenta em razão de a prefeitura realizar a coleta de resíduos na área urbana, porém deixando a zona rural parcialmente sem coleta.

$\mathrm{O}$ valor alto do $\mathrm{I}_{\mathrm{CV}}$ se deve pelo fato de não existir ocorrência de casos de esquistossomose e leptospirose, sendo constatados poucos casos de dengue. Devido à má distribuição de renda encontrada no município, já que a maior parte da população sobrevive com menos de 3 salários mínimos, o $I_{S E}$, foi influenciado negativamente, por outros fatores igualmente importantes para este indicador, como o índice de alfabetização e mortalidade. Os dados obtidos também não favoreceram Santa Terezinha de Itaipu.

\section{São Miguel do Iguaçu}

O valor do $\mathrm{I}_{\mathrm{ES}}$, no município, indica que cerca de $50 \%$ da população local é atendida pelo serviço de coleta e tratamento de esgoto. Já o valor numérico encontrado para $\mathrm{I}_{\mathrm{RS}}$ se dá devido ao fato de que a empresa que realiza o trabalho de coleta não atende toda a área rural, mesmo atendendo a totalidade da área urbana.

$\mathrm{O}$ valor encontrado para o $\mathrm{I}_{\mathrm{CV}}$, justifica-se pelo fato de não terem sido constatados casos de esquistossomose e leptospirose, porém houveram alguns registros de dengue pela secretaria da saúde. $\mathrm{O}$ resultado para o $\mathrm{I}_{\mathrm{RH}}$ deve-se pelo fato de o município possuir vários córregos e riachos disponíveis para abastecimento. $\mathrm{O} \mathrm{I}_{\mathrm{SE}}$ que se relaciona à saúde pública, renda e educação, foi encontrado como um valor mediano, e isso ocorreu devido ao número de pessoa analfabetas e a baixa renda da maior parte da população do município. 


\section{Itaipulândia}

No que se refere ao $\mathrm{I}_{\mathrm{ES}}$, os valores baixos encontrados devem-se à ausência de coleta de metade do esgoto que, por consequência, não é tratado. $\mathrm{OI}_{\mathrm{RS}}$ recebeu esta pontuação por meio do atendimento tanto urbano quanto rural que a prefeitura disponibiliza para a coleta de resíduos.

$\mathrm{O}$ controle de vetores obteve o menor valor entre os indicadores do município, devido ao fato de que o mesmo possuiu, casos de dengue e de leptospirose nos últimos 5 anos. Se tratando dos $\mathrm{I}_{\mathrm{RH}}$ o município é abastecido tanto pelo lago de Itaipu quanto por poços. O $\mathrm{I}_{\mathrm{SE}}$ resulta do fato de a renda familiar ser melhor que nos demais municípios e ainda a população possuir uma taxa maior de alfabetização.

\section{Matelândia}

Para os serviços de coleta e tratamento de esgoto há um há um atendimento de pouco menos de 50\% da população local, o restante dos habitantes utiliza o método da fossa séptica, porém, estima-se que nos próximos anos, essa porcentagem seja maior. $\mathrm{O} \mathrm{I}_{\mathrm{RS}}$ obteve tal valor, pela capacidade do município em suprir as necessidades da população tanto urbana quanto rural na coleta do resíduo.

Já o $\mathrm{I}_{\mathrm{CV}}$ conseguiu um menor valor devido ao município conter casos registrados de dengue e leptospirose nos últimos 5 anos, fazendo com que a média do indicador obtivesse uma queda. $\mathrm{O} \mathrm{I}_{\mathrm{RH}}$, se deu pelo fato de o município possuir quatro poços para abastecer a população local, além de poder contar, se necessário for, com os municípios vizinhos. Quanto ao $\mathrm{I}_{\mathrm{SE}}$, o mesmo não obteve um valor maior devido a renda média do município.

\section{Medianeira}

Medianeira possui o sistema de coleta e tratamento de esgoto, atendendo 1.528 domicílios; os demais 14.385 utilizam o método da fossa séptica. $\mathrm{O} \mathrm{I}_{\mathrm{RS}}$ se deu pelo fato de a empresa responsável pela coleta de lixo operar tanto na zona rural quanto na urbana. Não foram constatados casos de esquistossomose e leptospirose nos últimos cinco anos no município. Tratando-se dos recursos hídricos, a população conta com um poço para o abastecimento e com os municípios vizinhos se necessário for. $\mathrm{O} \mathrm{I}_{\mathrm{SE}}$, se deu principalmente pela renda média dos habitantes do município.

\section{Céu Azul}

Em Céu Azul o $\mathrm{I}_{\mathrm{ES}}$ obteve valor alto (Tabela 5) pois os órgãos responsáveis conseguem atender todos os habitantes além de tratar todo o esgoto coletado da cidade que por média é um valor de $25.957 \mathrm{~m}^{3} /$ mês. $\mathrm{O} \mathrm{I}_{\mathrm{RS}}$ obteve um valor menor que os demais indicadores, pelo fato de o poder público municipal atender $100 \%$ da área urbana e não conseguir suprir a área rural que conta com 25 comunidades. O que a prefeitura faz é estipular um ponto onde os moradores rurais possam levar seus resíduos sólidos, porém poucos dispõem adequadamente seus resíduos. 
Foram obtidos valores médios para o $\mathrm{I}_{\mathrm{CV}}$ visto que o município não constatou infestação de Aedes aegypti nem casos de esquistossomose, porém, o município apresentou casos de leptospirose o que interferiu neste indicador. Tratando-se do $\mathrm{I}_{\mathrm{RH}}$, o munícipio é atendido pelos municípios vizinhos, principalmente Cascavel. $\mathrm{O} \mathrm{I}_{\mathrm{SE}}$ se dá pelo fato de que apenas 600 habitantes, de um total de 11.032, são analfabetos, além de, dentre os municípios estudados.

\section{Cascavel}

Em Cascavel pode-se observar que o $\mathrm{I}_{\mathrm{RH}}$, que tem por finalidade quantificar a demanda em relação aos mananciais, apresentou seu valor pelo fato de que são utilizados 15 poços para abastecer a cidade, fora os mananciais principais que auxiliam no abastecimento do município sendo estes o Rio Cascavel, Rio Peroba e Rio Saltinho.

$\mathrm{O} \mathrm{I}_{\mathrm{CV}}$ chegou a esse resultado, pois o município apresentou alguns casos confirmados de leptospirose, embora nenhum caso de esquistossomose tenha sido catalogado, porém há confirmação de 396 casos de dengue na cidade. $\mathrm{O} \mathrm{I}_{\mathrm{RS}}$ teve uma pontuação alta, o que se deu devido ao fato de que as empresas de coleta de resíduos conseguem atender toda a população.

$\mathrm{O}$ indicador de esgoto sanitário foi alcançado, devido à coleta e tratamento de esgoto sanitário do município atingirem a totalidade do gerado. $\mathrm{O} \mathrm{I}_{\mathrm{SE}}$ alcançou uma pontuação que se justifica pelos dados levantados que mostram que apenas $6,6 \%$ da população da cidade não contém nenhum estudo e $41,64 \%$ da população recebe menos do que três salários mínimos.

\section{Consumo de energia elétrica dos municípios estudados}

Segundo Garcez (1976), os fatores que evidenciam o consumo de energia estão interligados às características da população, como renda e educação, e ainda, 2,5\% do consumo total de energia elétrica do país são consumidos pelos prestadores de serviços de distribuição de água e esgotamento sanitário cuja quantia equivale a 9,8 bilhões de $\mathrm{kWh} /$ ano. Devido ao crescimento populacional, serviços de saneamento básico deverão ser ampliados de acordo com o autor.

Para Achão (2003), as diferenças de classes sociais estão vinculadas ao consumo de energia elétrica, desta forma, as pessoas mais favorecidas buscam conforto e, por consequência, acabam consumindo quantidade maior de energia. Garcez (1976) afirma, também, que o número de indústrias pode vir a influenciar no consumo de energia elétrica de uma região.

A Tabela 5 é importante para as análises vindouras, pois determina se existe ou não diferença estatística, entre as médias per capta de consumo de energia elétrica de cada munícipio. 


\section{Tabela 5 - Média per capita do consumo de energia de cada município de 2008 a 2012}

\begin{tabular}{|c|c|c|}
\hline & Município & $\begin{array}{l}\text { Média per capta } \\
\left(\text { Mwh.ano.hab }{ }^{-1}\right)\end{array}$ \\
\hline \multirow{4}{*}{ 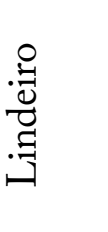 } & Foz do Iguaçu & $4,99020 \mathrm{ef}$ \\
\hline & Santa Terezinha de Itaipu & $4,83820 \mathrm{f}$ \\
\hline & São Miguel do Iguaçu & $5,96540 \mathrm{cdef}$ \\
\hline & Itaipulândia & $7,15740 \mathrm{~cd}$ \\
\hline \multirow{4}{*}{ Z } & Matelândia & $12,28700 \mathrm{a}$ \\
\hline & Medianeira & $7,34180 \mathrm{c}$ \\
\hline & Céu Azul & $9,34560 \mathrm{~b}$ \\
\hline & Cascavel & 5,70840 def \\
\hline
\end{tabular}

*As médias seguidas de mesma letra na coluna, não diferem estatisticamente entre si pelo teste de Tukey a 5\% de probabilidade

Na Tabela 5, é possível verificar que os municípios de Matelândia e Céu Azul se destacam no consumo per capto, seguidos por Medianeira, Itaipulândia e São Miguel do Iguaçu. As menores médias per captas ocorreram, em maioria, nos municípios com maiores números de habitantes (IBGE, 2013).

É possível constatar, na Tabela 4, que os municípios de Foz do Iguaçu, Cascavel, São Miguel do Iguaçu e Santa Terezinha de Itaipu obtiveram os menores valores para o $\mathrm{I}_{\mathrm{SE}}$. Nota-se que este valor justifica a quantidade consumida de energia por estes municípios (Tabela 5) já que nestes, o índice de pobreza é maior que os demais municípios assim como o índice de pessoas sem qualquer grau de escolaridade. O autor Achão (2003) relata que classes menos favoráveis financeiramente têm tendência de menor consumo, o que justificaria o menor consumo destes municípios.

Embora no município de Itaipulândia o $\mathrm{I}_{\mathrm{SE}}$ (Tabela 4) tenha apresentado valor elevado, nota-se que o nível de consumo não acompanhou de forma similar ao que ocorreu nos demais municípios. Isso se dá devido ao fato de Itaipulândia possuir menor número de empreendimentos e comércios.

$\mathrm{O} \mathrm{I}_{\mathrm{SE}}$ para Medianeira foi um dos valores mais baixos, como mostra a Tabela 4, porém, nota-se, no que se refere ao consumo de energia elétrica, o município teve um índice mediano. Os municípios de Matelândia e Céu Azul atingiram valores mais elevados que os demais municípios para o $\mathrm{I}_{\mathrm{SE}}$, que justifica seu maior consumo de energia de acordo com o pensamento de Achão (2003).

O fato de Matelândia contar com um complexo industrial de aves e carnes, desde o ano de 1999, e que, no ano de 2012, o mesmo empreendimento começou a atender o mercado internacional (destinando a produção à exportação principalmente para a Europa e Ásia), justifica-se o aumento no consumo de energia elétrica nos anos de 2011 e 2012.Já o município de Céu Azul possui indústrias de linha seca, que efetuam o empacotamento de alimentos como pipoca, lentilha e sagu entre outros produtos não alimentícios (COOPERATIVA INDUSTRIAL LAR, 2014).

Assim, pode-se afirmar que, embora o indicador sócio-econômico esteja interligado ao consumo de energia elétrica conforme o raciocínio de Achão (2003), deve-se levar em consideração a influência de comércios e indústrias dos municípios em estudo, como afirmado por Garcez (1976), Santos (2016) e Teixeira (2017) 


\section{Consumo de água dos municípios em estudo}

Para Garcez (1976), algumas das características que podem influenciar no consumo de água são, além das condições climáticas, os hábitos higiênicos, situação econômica, educação sanitária, características do abastecimento local. Outras características citadas pelo autor, que podem aumentar o consumo de água em uma região é a presença de industrias e, até mesmo, o fato de sua região estar em desenvolvimento. Visando à comparação entre o consumo de água per capita em cada um dos municípios estudados, a Tabela 6 possui os dados, bem como a análise estatística, referentes ao volume médio consumido por habitante na área de estudo.

\section{Tabela 6 - Média per capita de consumo de água nos municípios estudados de 2008 a 2012}

\begin{tabular}{|c|c|c|}
\hline & Município & $\begin{array}{l}\text { Média per capita } \\
\left(\mathrm{m}^{3} \text {.ano.hab }{ }^{-1}\right)\end{array}$ \\
\hline \multirow{4}{*}{ 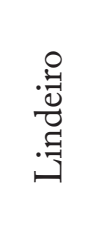 } & Foz do Iguaçu & $47.85275 \mathrm{a}$ \\
\hline & Santa Terezinha de Itaipu & $43.46190 \mathrm{ab}$ \\
\hline & São Miguel do Iguaçu & $34.02508 \mathrm{bc}$ \\
\hline & Itaipulândia & 31.29865 c \\
\hline \multirow{4}{*}{ 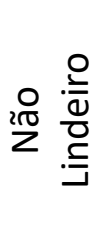 } & Matelândia & $38.85677 \mathrm{abc}$ \\
\hline & Medianeira & $43.13770 a b$ \\
\hline & Céu Azul & $39.78980 \mathrm{abc}$ \\
\hline & Cascavel & $42.45544 a b$ \\
\hline
\end{tabular}

*As médias seguidas de mesma letra na coluna, não diferem estatisticamente entre si.

$\mathrm{Na}$ Tabela 6,é possível verificar que, embora o consumo seja pouco divergente nos municípios estudados, houve menor consumo de água nos municípios de Itaipulândia e São Miguel. Um fato que deve ser destacado, é que no primeiro, $\mathrm{I}_{\mathrm{SE}}$ foi elevado, o que diverge do raciocínio de Achão (2003); já no segundo, embora exista presença de agroindústrias, o $\mathrm{I}_{\mathrm{SE}}$ encontrado foi bastante baixo, o que poderia justificar tal índice de consumo.

De acordo com Garcez (1976), as presenças de indústrias nos municípios são fatores que influenciam no consumo da água. Cabe citar o volume gasto por alguns dos empreendimentos, com maior consumo de água, presentes nos municípios:

o Presidiários 454 litros/dia;

- 38 litros/dia/funcionário de presidiário;

o Hotel 181 litros/dia/cliente

o 38 litros/dia/empregado;

o Matadouro de pequeno porte 150 litros/dia/cabeça abatida;

o Escolas onde cada aluno consome 57 litros/dia e shopping center 38 litros/dia/ funcionário.

Sabendo da importância, no computo do consumo, destes empreendimentos, a Tabela 7 foi elaborada para constatar o número de cada um dos estabelecimentos presentes nos municípios estudados. Observa-se que alguns dos municípios, que possuem maior número de empreendimentos de grande consumo, são os mesmos que, na tabela 5 apresentam maior 
volume per capto consumido, exceto por São Miguel do Iguaçu, que possui grande consumo embora não possua muitos empreendimentos, mas isso provavelmente ocorre devido ao tipo de empreendimento presente no município.

\section{Tabela 7 - Número de estabelecimentos com grande demanda de água, nos municípios.}

\begin{tabular}{ccccccc}
\hline Municípios & Escolas & Hotéis & Prisões & Shoppings & Matadouros & $\begin{array}{c}\text { Empresas } \\
\text { atuantes }\end{array}$ \\
\hline Foz do Iguaçu & 520 & 70 & 2 & 1 & 0 & 9.152 \\
Santa Terezinha de Itaipu & 42 & 1 & 0 & 0 & 2 & 818 \\
São Miguel do Iguaçu & 68 & 2 & 0 & 0 & 2 & 1.020 \\
Itaipulândia & 24 & 2 & 0 & 0 & 1 & 396 \\
Matelândia & 44 & 1 & 0 & 0 & 2 & 537 \\
Medianeira & 90 & 1 & 0 & 0 & 2 & 1.874 \\
Céu Azul & 30 & 0 & 0 & 0 & 2 & 336 \\
Cascavel & 578 & 7 & 2 & 4 & 0 & 13.063 \\
\hline
\end{tabular}

Fonte: IBGE (2010).

Seguindo o raciocínio de Garcez (1976), sobre a relação entre indústrias e consumo, o município de Itaipulândia possui grandes quantidades de pecuária suína, contando com duas unidades produtoras de Leitões. Matelândia por sua vez, possui um frigorífico que abate em média 270 mil aves por dia.

Os municípios de Medianeira e São Miguel do Iguaçu contam com industrias de ração, e, finalmente Céu Azul, município onde existe uma indústria produtora de óleo e farelo de soja, sendo que começou a atender o mercado nacional em 2012. Tais fatos podem justificar o aumento no consumo de água nestes municípios nos anos de 2011 e 2012.

Os maiores consumidores de água foram, além de Medianeira, os municípios de Foz do Iguaçu e Cascavel. Isso se esclarece devido (além dos motivos já citados) à perda de água nos sistemas de distribuição. Foi constatado que o município de Foz do Iguaçu perde aproximadamente 310 litros por dia em cada ligação; em Cascavel, esse valor é de aproximadamente 250 litros. Essa problemática, ocorre devido às ligações clandestinas, vazamentos ou erros de ligações. Estes problemas causam indiretamente impactos ambientais, já que na falta de água, inicia-se a busca por mais mananciais para o abastecimento (ABES, 2013).

Tratando-se das condições climáticas que também é dada como uma das características que influenciam no consumo de água, Barbiero e Lemos (2011) contradiz Garcez (1976), apresentando resultados que demonstram que as alterações climáticas regionais não podem ser relacionadas com variações no consumo de água, desta maneira, também não pôde ser utilizas como parâmetro para o consumo de abastecimento de água. Pode-se dizer, portanto, que as variáveis, condição climática, características sociais e econômicas, devido às semelhanças entre os municípios estudados, não serviram como parâmetro avaliativo para o volume consumido de água, já que todos pertencem à mesma região geográfica e à mesma bacia hidrográfica. 


\section{Conclusões}

1. O ISA depende exclusivamente da fonte de dados de uso, mas com a metodologia de Piza, foi possível encontrar valores numéricos que indicam a salubridade dos municípios estudados.

2. Os royalties não têm interferência significativa na salubridade dos municípios estudados.

3. O consumo de água em cada município foi dependente dos empreendimentos instalados em nível local.

4. O ISA apresentou-se como uma ótima ferramenta para definição da salubridade ambiental dos municípios em estudos, com potencial de uso para monitoramento e de gestão ambiental.

\section{Referências}

ACHÃO, C. C. L. Análise da estrutura de Consumo de Energia Elétrica pelo Setor Residencial Brasileiro. 2003. p. 122, Tese (Doutorado em Engenharia) - Universidade Federal do Rio de Janeiro, Rio de Janeiro, 2003.

ABES. Associação Brasileira de Engenharia Sanitária e Ambiental. Disponível em < http://www.abes-sp.org.br/arquivos/perdas_resumo.pdf>. Acesso em 12 Out. 2014.

AKERMAN, M.; STEPHENS, C.; CAMPANARIO, P.; MAIA, B. B. Saúde e meio ambiente: uma análise de diferenciais intra-urbanos enfocando o Município de São Paulo, Brasil. Revista Saúde Pública, São Paulo, v. 28, n. 4, p. 320-325, 1994.

BARBIERO, L. C. S; LEMES, S. L. A influência das estações do ano no consumo de água em Maringá- PR. Revista Percurso, v. 3, n. 1, p. 183 - 191, 2011.

FUNASA. Fundação Nacional de Saúde. Manual de saneamento, 2006. Disponível em: http://www.funasa.gov.br/internet/arquivos/biblioteca/eng/eng_saneam.pdf >.Acesso em: 01 mar. 2012.

GARCEZ, L. N. Elementos de engenharia hidráulica e sanitária. 2.ed. São Paulo: Editora Atena, 1976.

IBGE. Instituto Brasileiro de Geografia e Estatístico. Disponível em: http://cidades.ibge. gov.br/painel/painel.php?lang=\&codmun=411560\&search=parana $\mid$ matelandia $\mid$ infografi cos:-dados-gerais-do-municipio>. Acesso em 06 de dezembro de 2013.

LEISMANN, E. L. Os Royalties de Itaipu nos Municípios lindeiros ao lago e no Estado do Paraná, Ciências Sociais Aplicadas, v. 6, n. 11, p. 1-12, 2006.

REIS, M. F. dos; OLIVEIRA, R. F. de. A Relação Planejamento urbano e qualidade de vida: Análise sobre a cidade de Campinas - SP, Brasil. Revista Geográfica de América Central, Costa Rica, v. 2, p.1-12, jul.2011. Disponível em: http://www.revistas.una.ac.cr/ index.php/geografica/article/view/2125>. Acesso em: 22 mar. 2014. 
SILVA, N. V. S. As condições de Salubridade Ambiental das Comunidades Periurbanas da Bacia do Baixo Gramame: Diagnóstico e Proposição de Benefícios. 2006. p. 150, Dissertação (Mestrado em Engenharia Urbana) - Universidade Federal da Paraíba, João Pessoa, 2006.

PINTO, L.P.; MARI, A.C. C.; MARI JUNIOR, A.; AZEVEDO, K. D.; CABRAL, C.; FRIGO, E. P. Condição ambiental do município de Diamante do Oeste - PR, Brazilian Journal of Biosystems Engineering, v. 10, n. 1, p. 62-68, 2016.

PIZA, F. J. T. Indicador de salubridade ambiental. In: SEMINÁRIO SOBRE INDICADORES DE SUSTENTABILIDADE, 2000. São Paulo. Anais ... São Paulo: Editora Atena, 2000.

PREFEITURA, Foz do Iguaçu. Disponível em: http://www.pmfi.pr.gov.br/>. Acesso em 16 Out. 2013.

PREFEITURA, Santa Terezinha de Itaipu. Disponível em: http://www.stitaipu.pr.gov. br/>. Acesso em 15 de Set. 2013.

PREFEITURA, São Miguel do Iguaçu. Disponível em:< www.saomiguel.pr.gov.br> . Acesso em 21 de agosto de 2013

PREFEITURA, Itaipulândia. Disponível em: http://www.itaipulandia.pr.gov.br/ conheca>. Acesso em 05 de dezembro de 2013.

PREFEITURA, Matelândia. Disponível em: http://www.matelandia.pr.gov.br/ >. Acesso 14 de Mar. 2013.

PREFEITURA, Medianeira. Disponível em: < http://www.medianeira.pr.gov.br/>. Acesso em: 19 de fevereiro de 2013.

PREFEITURA, Céu Azul. Disponível em: http://www.ceuazul.pr.gov.br/portal1/intro. asp?iIdMun=100141075 > . Acesso 14de Maio de 2013.

PREFEITURA, Cascavel. Disponível em: http://www.cascavel.pr.gov.br/indicadores. php.>. Acesso em 27 fev. 2013.

SANTOS, F. F. S. Adaptação do indicador de salubridade ambiental (ISA) para análise do saneamento básico na cidade de Brejo Grande/SE. 2016. p. 161, Dissertação (Mestrado em Desenvolvimento e Meio Ambiente) - Universidade Federal de Sergipe, 2016.

SANTOS, R. F.; CABRAL, A. C.; FRIGO, E. P.; BASTOS, R. K.; PLACIDO, H. F.; PINTO, L.P. Aplicação de indicadores no município de Palotina - PR, Brazilian Journal of Biosystems Engineering, v. 9, n. 1, p. 84-89, 2015. 
TEIXEIRA, D. A. Construção e determinação do indicador de salubridade ambiental (ISA/OP) para as áreas urbanas do município de Ouro Preto, MG. 2017. p. 171. Dissertação (Mestrado em Engenharia Ambiental) - Universidade Federal de Ouro Preto, 2017.

WORLD HEALTH ORGANIZATION (WHO). The Urban Health Index - A handbook for its calculation and use. Kobe, Japão, 2014. 